\title{
The challenge given by Zika virus
}

\author{
Juan Sebastian Vargas Rodriguez
}

Public health has been a great challenge in many parts of the world, and underdeveloped nations present the greatest challenges in addressing the health problems in the field of public health. In recent years there have been outbreaks of reemerging diseases transmitted by vectors, such as the mosquito, Zika virus disease belonging to this group.

Zika virus, which was discovered incidentally in Uganda in 1947 in the course of monitoring mosquitoes and primates, had remained a dark virus confined to a narrow equatorial belt that traversed Africa and Asia [1]. Arboreal mosquitoes such as Aedes africanus and rarely caused infections known as "spillage" in humans, even in highly enzootic areas [1].

The challenges given by Zika virus began from its transmission, constituting an evolution of this virus in this aspect. The arboreal Aedes aegypti then adapted to deposit its eggs in domestic containers containing water and to feed on humans, which led to the adaptation of arboreal viruses to infect humans [2].

Although transmission is caused mainly by mosquitos of Aedes species, two instances of sexual transmission of Zika virus have been reported, both involving symptomatic males. No sexual transmission of Zika virus from infected women to their partners and from infected people without symptoms has been reported [3].

A high load of replicative Zika virus and Zika virus RNA was detected in semen samples, but Zika virus remained undetectable by real-time reverse transcription PCR in blood samples collected at the same time. These results suggest that viral replication may have occurred in the genital tract, but it is unclear when this replication began and how long it lasted [4]. In a recent study on sexually transmitted routes involving experiments on mice, viral replication occurs in the brain and spleen along with viral load in vaginal washings 10 days after infection. The study presented a transgenital transmission accompanied by a variation in the levels of hormones of the female reproductive tract with a prolonged prevalence of viral replication [5].

Another study noted that transmission goes beyond that by vectors or the parenteral route. Viable Zika virus particles were isolated from saliva and urine collected from two acutely infected people, and therefore they were recognized as vectors for transmission [6].

In addition to the evolutionary challenge in its transmission, Zika virus has brought with it greater controls and vigilance with regard to pregnant women. A series of reports suggesting an increase in the number of babies born with microcephaly in areas affected by Zika virus began to emerge, and Zika virus RNA was identified in the amniotic fluid of two women whose fetuses had microcephaly according to findings from ultrasonography performed before birth [7].
CORRESPONDING AUTHOR:

Juan Sebastian Vargas Rodriguez Medical Program, Investigation Department, Simon Bolivar University, Barranquilla, Colombia E-mail: vargassebastian152@ gmail.com

Orcid: 0000-0002-6698-5864

Received 17 June 2018; Accepted 11 July 2018 
The Ministry of Health of Brazil established a working group to investigate the possible link between microcephaly and Zika virus infection during pregnancy, and to create a register of microcephaly cases (head circumference equal to or greater than two standard deviations below the mean for sex and gestational age at birth) and for the outcomes of pregnancies of women who may have been infected with Zika virus during pregnancy [7].

From a cohort of 35 babies with microcephaly who were born between August and October 2015 in eight of the 26 states of Brazil and who were documented in the registry whose mothers (35) had lived in or visited areas affected by Zika virus during pregnancy, 25 babies (71.0\%) had severe microcephaly, 17 (49.0\%) had at least one neurological abnormality, and 27 underwent neurological imaging studies, all of whom had abnormalities. Test results for other congenital infections were negative [7].

Garcez et al. used human neural stem cells, neurospheres, and brain organoids to show that Zika virus, but not the flavivirus-related dengue virus, induces cell death in neural stem cells, disrupts the formation of neurospheres, and reduces the growth of organoids. The Zika virus-infected neurospheres had apoptotic nuclei and contained virus particles that bound to the membranes and were observed both in the mitochondria and in the vesicles of the cells within the infected neurospheres [8].

Using a similar system of brain organoids derived from embryonic stem cells, they demonstrated that Zika virus can infect these cells, which resulted in a decrease in the size of the organoids, and the size of the organoids was correlated with the viral copy number. They also found that Zika virus infection affected the innate immune receptor Toll-like receptor 3 (TLR3), which was previously implicated in neuroinflammatory and neurodegenerative conditions [9].

TLR3 protein decreased the phenotypic effects of viral infection. A pathway analysis of changes in gene expression during activation of TLR3 showed that 41 genes were related to neuronal development, indicative of a potential mechanistic connection to interrupted neurogenesis [10].

It is not known if sexual transmission of Zika virus poses a different risk for congenital infection than that of transmission by mosquitos [3].
It should be borne in mind that it is more complicated for this disease and is more serious than the virus of infection by the virus. It is possible that the symptoms are nonspecific and can be confused with other diseases such as chikungunya and the virus caused by the arboviral virus. differential diagnosis plays a vital role in regions where the virus circulates in conjunction with other arboviruses and therefore, Zika virus infection can be misdiagnosed. Asymptomatic individuals can be reservoirs for the transmission of the virus and have unclear diagnoses [5].

In accordance with the recent guidelines of the Centers for Disease Control and Prevention (CDC) to assess pregnant women who may be exposed to the Zika virus, it was necessary to evaluate asymptomatic pregnant women as long as there are sufficient laboratory facilities available. The most specific diagnostic approach is the molecular amplification of serum samples and that ideal test method for Zika virus infection in the acute phase of the disease. However, serological tests are not recommended, since Zika virus IgM could be undetectable during the acute phase [5].

An expanding spectrum of fetal malformations associated with intrauterine Zika virus infection continues to be described. Currently known as congenital Zika syndrome (CZS), this new congenital malformation syndrome includes not only microcephaly and fetal brain damage, but also a range of developmental abnormalities, including the locomotor, ocular, craniofacial, genitourinary, pulmonary, and other manifestations [7]. This means that the challenge is even greater and that surveillance by health organizations should be more rigorous in the face of any eventuality such as outbreaks that may endanger pregnant women as well as developing fetuses.

The presence of Aedes mosquitos enables Zika virus to invade new areas and impose a worldwide risk as there are no preventive approaches or vaccines for Zika virus infection. The control measures depend on the eradication of mosquito vector breeding foci. Methods that sustain the environment and preserve the effectiveness should be used for efficient control of Zika virus. With the advent of high-level research, an effective and safe Zika virus vaccine will probably be developed soon. An initiative for the development of a Zika 
virus vaccine was started by National Institutes of Health in 2015 [5].

\section{References}

1. Pierson TC, Diamond MS. Flaviviruses. Fields virology. 6th ed. Vol. 1. Philadelphia: Wolters Kluwer; 2014. pp. 746-94.

2. Fauci A, Morens D. Zika virus in the Americas - yet another arbovirus threat. N Engl J Med 2016;374:601-4.

3. Hills SL, Russell K, Hennessey M, Williams C, Oster AM, Fischer $\mathrm{M}$, et al. Transmission of Zika virus through sexual contact with travelers to areas of ongoing transmission - continental United States, 2016. MMWR Morb Mortal Wkly Rep 2016;65:215-6.

4. Musso D, Roche C, Robin E, Nhan T, Teissier A, Cao-Lormeau VM. Potential sexual transmission of Zika virus. Emerg Infect Dis 2015;21(2):359-61.

5. Shankar A, Patil A, Skariyachan S. Recent perspectives on genome, transmission, clinical manifestation, diagnosis, therapeutic strategies, vaccine developments, and challenges of Zika virus research. Front Microbiol 2017;8:1761.
6. Silva LR, Souza AM. Zika virus: what do we know about the viral structure, mechanisms of transmission, and neurological outcomes? Rev Soc Bras Med Trop 2016;49:267-73.

7. Schuler-Faccini L, Ribeiro EM, Feitosa IM, Horovitz DD, Cavalcanti DP, Pessoa A, et al. Possible association between Zika virus infection and microcephaly - Brazil, 2015. MMWR Morb Mortal Wkly Rep 2016;65:59-62.

8. Garcez PP, Loiola EC, Madeiro da Costa R, Higa LM, Trindade P, Delvecchio R, et al. Zika virus impairs growth in human neurospheres and brain organoids. Science 2016;352(6287):816-8.

9. Dang J, Tiwari SK, Lichinchi G, Qin Y, Patil VS, Eroshkin AM, et al. Zika virus depletes neural progenitors in human cerebral organoids through activation of the innate immune receptor TLR3. Cell Stem Cell 2016;19(2):258-65.

10. Alvarado MG, Schwartz DA. Zika virus infection in pregnancy, microcephaly, and maternal and fetal health. What we think, what we know, and what we think we know. Arch Pathol Lab Med 2017;141:26-32. 